\title{
Surface roughness in InGaAs channels of high electron mobility transistors depending on the growth temperature: Strain induced or due to alloy decomposition
}

\author{
F. Peiró, ${ }^{\text {a) }}$ A. Cornet, and J. R. Morante \\ EME, Enginyeria i Materials Electrònics, Departament Electrònica, Universitat de Barcelona, \\ Avda. Diagonal 647, 08028 Barcelona, Spain \\ M. Beck and M. A. Py \\ IMO, Institute of Micro and Optoelectronics, Department of Physics, Swiss Federal Institute of Technology, \\ CH-1015 Lausanne, Switzerland
}

(Received 5 January 1998; accepted for publication 10 March 1998)

\begin{abstract}
InAlAs/InGaAs/InP based high electron mobility transistor devices have been structurally and electrically characterized, using transmission electron microscopy and Raman spectroscopy and measuring Hall mobilities. The InGaAs lattice matched channels, with an In molar fraction of 53\%, grown at temperatures lower than $530{ }^{\circ} \mathrm{C}$ exhibit alloy decomposition driving an anisotropic InGaAs surface roughness oriented along [1 $\overline{1} 0$ ]. Conversely, lattice mismatched channels with an In molar fraction of $75 \%$ do not present this lateral decomposition but a strain induced roughness, with higher strength as the channel growth temperature increases beyond $490{ }^{\circ} \mathrm{C}$. In both cases the presence of the roughness implies low and anisotropic Hall mobilities of the two dimensional electron gas. (C) 1998 American Institute of Physics. [S0021-8979(98)01612-0]
\end{abstract}

\section{INTRODUCTION}

A basic research into the growth of layers in pseudomorphic high electron mobility transistor (HEMT) is of great interest in order to avoid well nonuniformities that could lead to a deterioration of the device performance. Concerning the In composition in the InGaAs channel and hence system mismatch, a three-dimensional (3D) growth mode can take place not only because of stress induced roughening in highly strained layers but also because of the alloy lateral selfdecomposition. Besides, the channel growth temperature $\left(T_{g}\right)$ is also critical for the determination of the onset of plastic relaxation and for the growth mode transition from two- (2D) to three-dimensional growth modes as a mechanism of strain accommodation. Low $T_{g}$ may allow to extend the critical thickness range, because of thermodynamics criteria, but high $T_{g}$ favors the kinetics of the process enhancing adatom migration, leading to a smooth growth front. The structural quality of InGaAs wells can also be affected by the presence of inhomogeneities in the InAlAs buffer layer which can propagate through the well. The origin of these inhomogeneities has been related to a mechanism of In segregation occurring during the growth of the buffer at relatively high $T_{g} \cdot 1$

In this work we have investigated by transmission electron microscopy (TEM) and Raman spectroscopy InAlAs/ InGaAs/InP HEMT heterostructures grown by molecular beam epitaxy (MBE). Our interest has been focused on the effects of channel growth temperature in the range $T_{g}$ $=470-530{ }^{\circ} \mathrm{C}$, for well compositions $x_{\mathrm{In}}=53 \%$ and $75 \%$ and well thickness $t_{w}=10 \mathrm{~nm}$ on the development of three-

\footnotetext{
${ }^{a)}$ Electronic mail: paqui@iris1.fae.ub.es
}

dimensional growth modes being strain induced or driven by InGaAs lateral decomposition. We correlate the twodimensional electron gas (2DEG) Hall mobility $(\mu)$ with TEM results to explain the degradation and anisotropy of the 2DEG mobilities.

\section{EXPERIMENTAL DETAILS}

The configuration of HEMT devices is from bottom to top: $400 \mathrm{~nm}$ of an InAlAs buffer layer over the InP substrate, a channel system consisting of $40 \mathrm{~nm}$ of a prechannel InGaAs layer (lattice matched) and an $\operatorname{In}_{x} \mathrm{Ga}_{1-x}$ As channel with thickness $t_{w}, 5 \mathrm{~nm}$ of an InAlAs spacer layer, a deltadoped Si donor layer $\left(6 \times 10^{12} \mathrm{~cm}^{-2}\right), 20 \mathrm{~nm}$ of an InAlAs Schottky barrier and finally, a cap layer of $10 \mathrm{~nm}$ undoped InGaAs. To study the effect of channel growth temperature $\left(T_{g}\right.$ ) on the electrical properties of the HEMTs, two series of HEMTs have been studied: the first set of samples have a channel system with a total thickness of $50 \mathrm{~nm}$ and completely lattice matched, whereas for the second set, the channel system consists of $40 \mathrm{~nm}$ of a lattice matched InGaAs subchannel and a strained well with $x_{\mathrm{In}}=75 \%$ and $t_{w}=10$ $\mathrm{nm}$. In both sets, the channels were grown in the range from 470 to $530{ }^{\circ} \mathrm{C}$.

The details of sample growth and HEMT electrical characterization have been described elsewhere. ${ }^{2}$ The 2DEG mobilities were investigated by Hall measurements carried out on bridge-shaped Hall bars along the [110] and [1 $\overline{10}]$ crystal directions. In the present work we correlate the anisotropy of Hall mobility with the presence of a surface roughness as investigated by TEM. TEM observations were carried out using a Hitachi H-800NA operated at $200 \mathrm{kV}$. Raman spectroscopy measurements have been done on beveled heterostructures to avoid problems of top layer absorption. Bevel- 


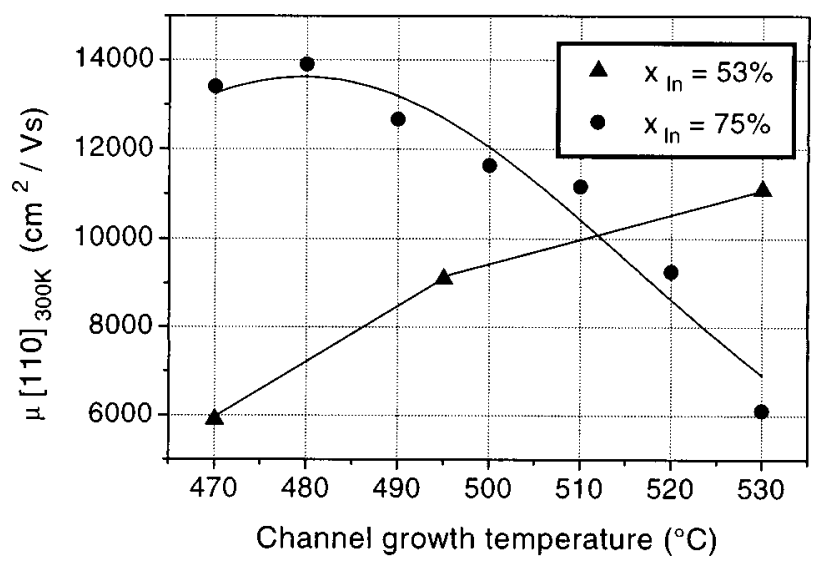

FIG. 1. Dependence of $\mu$ [110] on channel growth temperature in lattice matched and mismatched channels.

ing was made by mechanical polishing, using the polishing setup from a commercial SSM spreading resistance probe system. Pieces of the samples were mounted with wax on tilted holders having the desired bevel angle. Then, the samples were polished with fine diamond abrasive in an oilbased slurry and frosted glass plate. Since the bevel angle used was very low, just $0^{\circ} 34^{\prime}$, the polishing revealed as free surfaces the different layers of the heterostructure, with lateral dimensions wide enough for Raman spectra of the InGaAs channel to be obtained without a contribution from the InAlAs spacer layer or from the InAlAs buffer. The exposed surface of the InGaAs channel was $5 \mu \mathrm{m}$ wide, much larger than the $0.5 \mu \mathrm{m}$ of the laser probe. Room temperature Raman spectra were obtained using the $514.5 \mathrm{~nm}$ emission line of an Ar-ion laser in backscattering configuration.

\section{RESULTS AND DISCUSSION}

As shown in Fig. 1, the value of Hall mobility is strongly dependent on the growth temperature of the channel, both in lattice matched and in mismatched samples. In both cases, as has been reported in previous works, ${ }^{2,3}$ there is an anisotropic behavior in the mobility dependence, the $\mu$ [110] being more sensitive to the growth temperature and always presenting smaller values than in the perpendicular direction. However, there is a significant difference in the temperature influence: whereas the mobility of lattice matched samples increases with the channel growth temperature, it decreases in lattice mismatched samples. When correlating these results with the structural characteristics obtained by TEM techniques, an asymmetric distribution of stacking faults in the two $\langle 110\rangle$ slip systems is also noticeable, with a mean density $\rho_{\mathrm{SF}}$ around $10^{7} \mathrm{~cm}^{-2}$, whose origin is related to deficient substrate cleaning previous to growth. ${ }^{4}$ Nevertheless, as shown in Fig. 2, the temperature dependence of the stacking faults densities does not account for the evolution of the mobility values. Then, apart from the stacking faults, there must be another significant contribution to the reduction of the mobility. In effect, the plan-view observations of the two series of samples, shown in Figs. 3 and 4, also reveal the existence of an anisotropic and quasiperiodic surface undulation with hills and valleys alternating along the direction

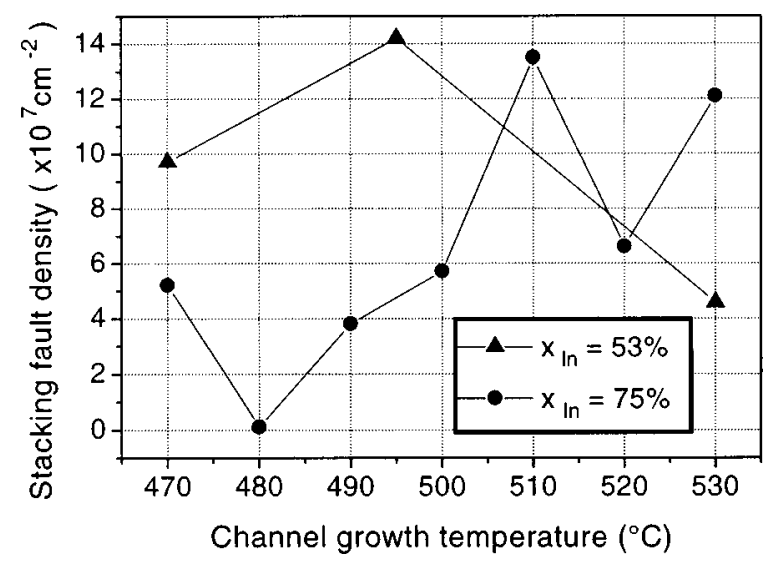

FIG. 2. Stacking fault density depending on the channel growth temperature.

[110] and the presence of a square-shaped contrast pattern oriented close to the $\langle 010\rangle$ directions. In the case of lattice matched samples (Fig. 3), the study of the dependence of the structural features with the temperature, show the following results: (i) the undulation becomes more evident as growth temperature decreases below $T_{g}=530{ }^{\circ} \mathrm{C}$. (ii) in the sample grown at the lowest $T_{g}$, the short periodicity of this undulation $(\approx 75 \AA)$ leads to a splitting of the diffraction spots on selected area diffraction patterns [see the inset of Fig. 3(a)],
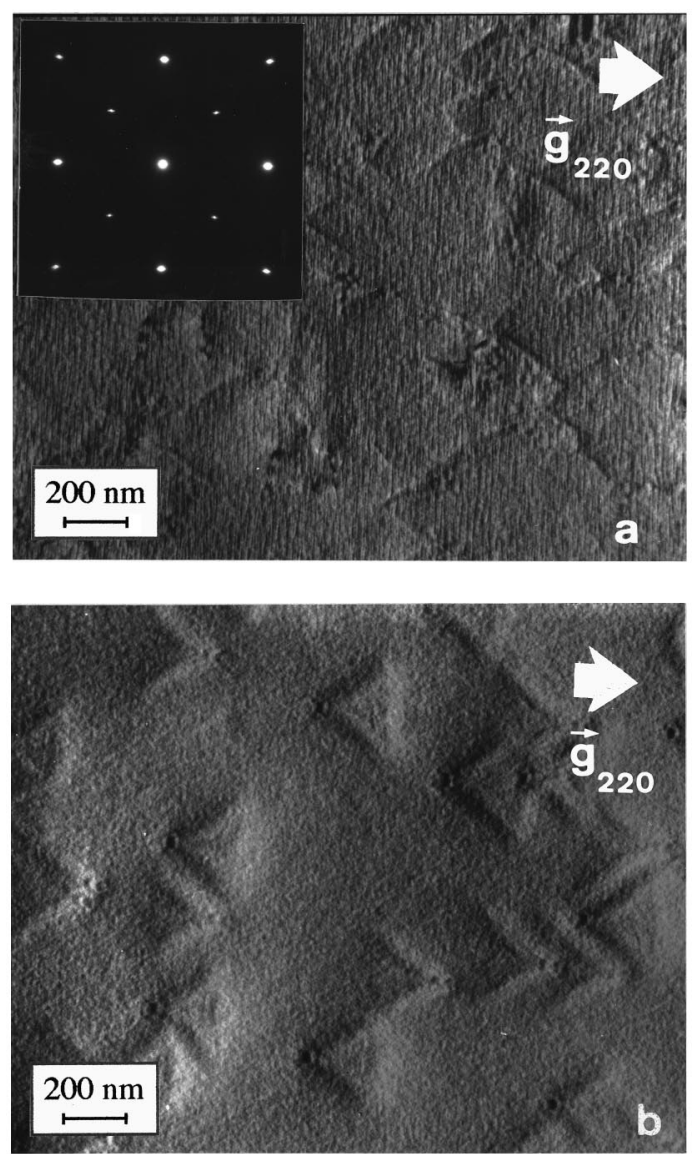

FIG. 3. (001) Plan-view images of samples with lattice matched InGaAs channel grown at (a) $470{ }^{\circ} \mathrm{C}$ exhibiting undulations alternating along [110], and (b) grown at $530^{\circ} \mathrm{C}$, without any undulations. 

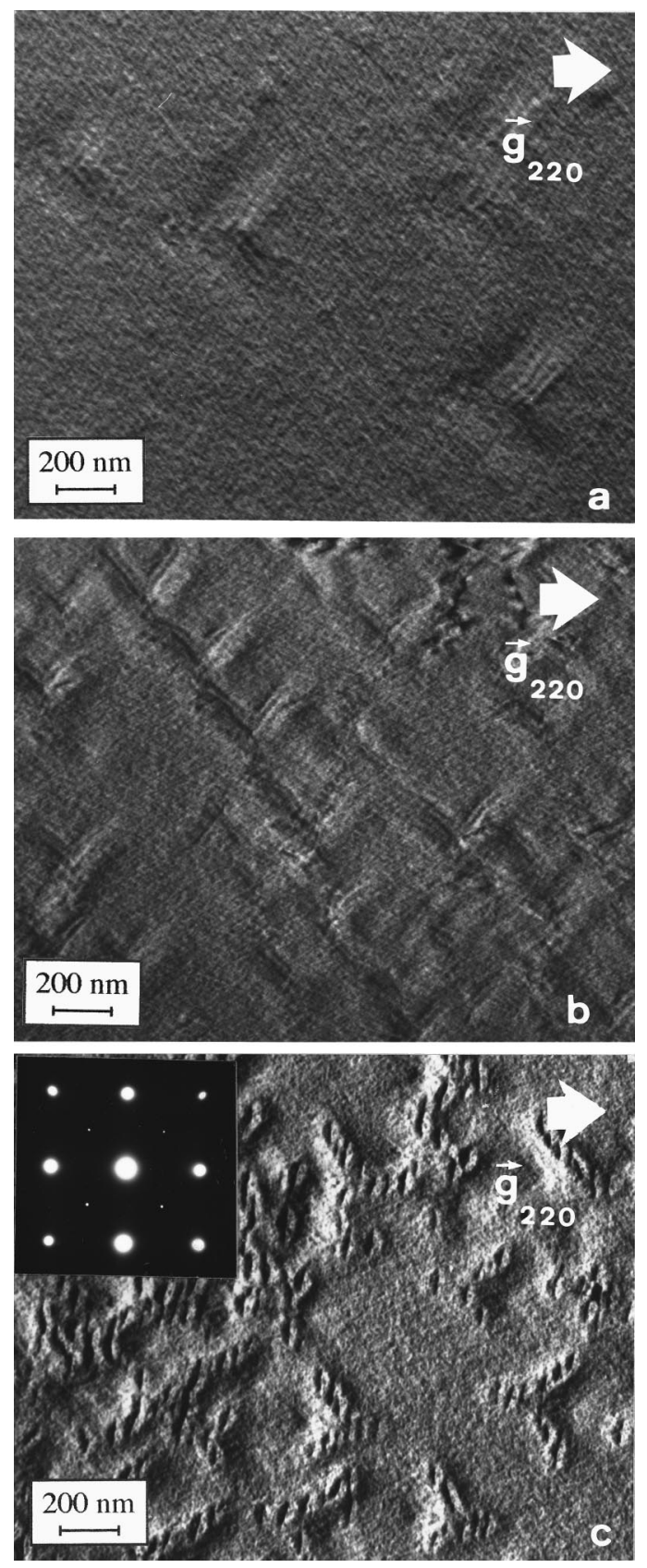

FIG. 4. (001) Plan-view images of samples with lattice mismatched InGaAs channel grown at (a) $480{ }^{\circ} \mathrm{C}$, (b) $500{ }^{\circ} \mathrm{C}$, and (c) $520^{\circ} \mathrm{C}$.

(iii) the appearance of the square-shaped contrast pattern does not depend on the channel growth temperature. The same study of temperature dependence was carried out on samples with lattice mismatched channel (Fig. 4), obtaining the following results: (i) higher strength of squared-shaped contrast as $T_{g}$ is increased; (ii) higher strength of anisotropic roughness (islands elongated to $[1 \overline{1} 0]$ ) as $T_{g}$ is increased, and preferentially linked to the coarse pattern or defect complexes. In both sets of samples, the evolution of surface roughness with the temperature follows the same relationship than the Hall mobilities one. Although the presence of the surface corrugation explains both the dramatic decrease of $\mu$ [110] but also the $\mu[1 \overline{1} 0]$ reduction, the different evolution of $\mu$ values with the temperature suggests that the origin of

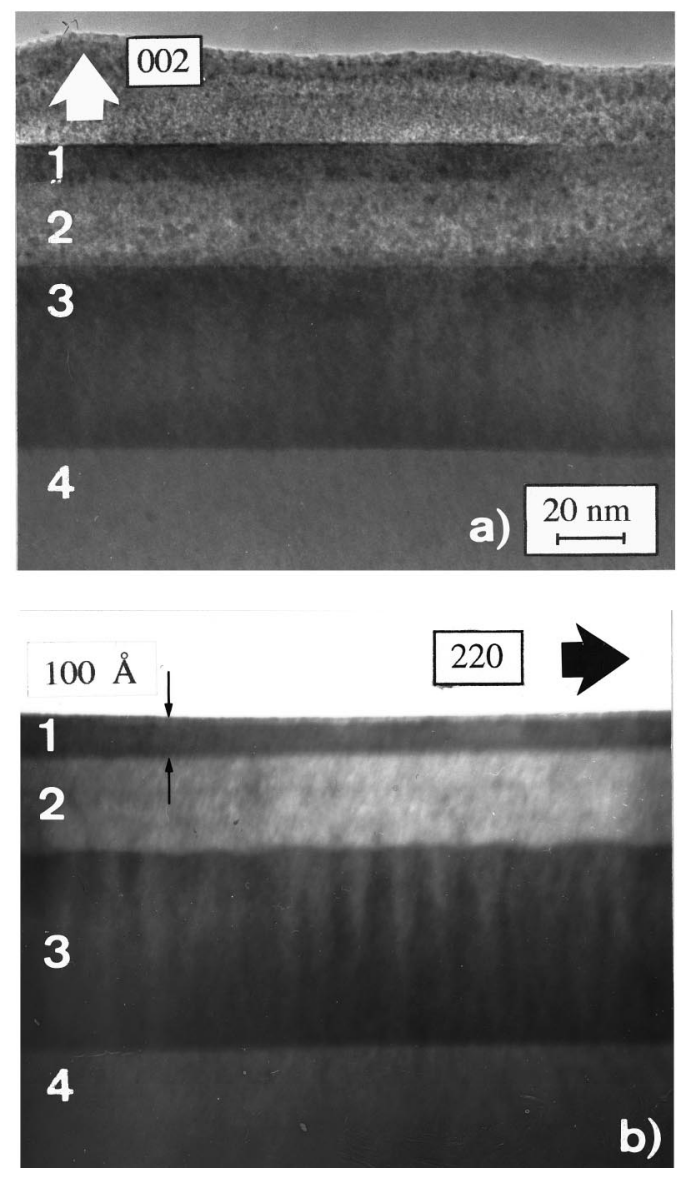

FIG. 5. $[1 \overline{10}]$ cross section of the samples with lattice matched channel grown (a) at $495{ }^{\circ} \mathrm{C}$, obtained with $g=002$ and (b) at $470{ }^{\circ} \mathrm{C}$, image obtained with $g=220$. In both cases lateral domains parallel to [001] are observable. The numbers correspond to: the InGaAs cap layer (1), the InAlAs donor and spacer layers (2), the channel and subchannel lattice matched InGaAs (3) and the InAlAs buffer layer (4).

the surface corrugation is different in lattice matched than in lattice mismatched samples. In order to further understand this origin, we have examined the samples in cross-section orientation.

\section{A. Surface corrugation in lattice matched samples}

XTEM images of the lattice matched samples grown at lower temperature (Fig. 5), show that there is a correspondence between the presence of lateral domains inside the InGaAs channel and the InGaAs top layer undulation, with dark domains correlated with surface valleys and bright domains with surface ripples. The origin of such lateral domains could be related to strain-induced lateral ordering extensively reported for strained layers of semiconductors. ${ }^{5,6}$ These previous studies have shown that the lateral composition modulation acts as a strain relieving mechanism that accommodates the mismatch between the epitaxial buffer layer and the substrate, depending on the growth temperature, substrate orientation, and layer-substrate mismatch. ${ }^{6}$ In the present work, however, we have some substantial differences. First of all, we are dealing with a lattice matched channel system, and therefore, the lateral modulation should not have developed as a strain relieving mechanism. Second, 
our samples have been grown on exact (001)-axis substrates, and hence, we do not have any steps at the interface that could develop the [001] columnar domains observed in XTEM images. Finally, in these samples, we have observed the lateral modulation with increasing strength in the layers grown at lower temperature, whereas for strained layers, the decomposition was found to be thermally activated. All these discrepancies can be explained if we take into account that the lattice matched samples, given the growth temperature and layer composition range, are expected to present spinodal decomposition due to the thermodynamically predicted immiscibility gaps for most of the ternary and quaternary III-V compounds. ${ }^{7}$ The spinodal decomposition arises from a balance between the entropy and enthalpy terms in order to reach the minimum Gibbs free energy of the system. When the compound is grown at low temperature, the enthalpy term dominates over the entropy term. Therefore, the system tends to reduce the state of bond strain separating the compound towards the respective binaries, and hence, reducing the total enthalpy term for alloy formation. Once the spinodal decomposition in In- and Ga-rich regions starts, the preferential segregation of the adatoms towards the compressive or tensile regions is responsible for the continuation of the decomposition during the subsequent growth. The higher growth rate in less strained regions, with respect to the underlying lattice parameter, should develop surface roughness during further growth. As $T_{g}$ increases, the entropy term becomes significant and then, a further reduction of the enthalpy is not essential to reduce the free energy.

This mechanism of spinodal decomposition explains the lateral modulation observed in our InGaAs lattice matched layers grown at this relatively low growth temperature $\left(T_{g}\right.$ $<500{ }^{\circ} \mathrm{C}$ ) and the absence of lateral decomposition in the layer grown at higher temperature $\left(T_{g}=530^{\circ} \mathrm{C}\right)$. In order to corroborate the presence of lateral composition domains, Raman spectroscopy measurements have been done on beveled heterostructures. This bevel was needed in order to overcome the problems related to light absorption of the cap and spacer layers when trying to work from the top of the HEMT. The exciting light penetration depth for these ternary compounds is around $\alpha^{-1}=40 \mathrm{~nm}$, and taking into account the thickness of the first layers, we would have obtained Raman spectra with very faint contribution of the region of interest. In order to test the possible contribution of the defects inside the layers to Raman diffusion, first of all we analyzed the InAlAs buffers of the three lattice matched layers. Raman spectra exhibit LO frequencies corresponding to the InAs- and AlAs-like peaks around 235 and $372 \mathrm{~cm}^{-1}$, respectively, without significant variations according to the defect distributions. Therefore, we assume that any possible differences of the InGaAs channel spectra are related to the presence of the lateral domains. These spectra are shown in Fig. 6(a). The dotted line below each spectra corresponds to the spectra fit and is shifted towards lower intensities for the purpose of clarity. Whereas in the channel grown at high temperature LO InAs- and GaAs-like peaks are defined with frequencies of 237 and $273 \mathrm{~cm}^{-1}$, respectively, there is a shift of these peaks towards higher (respectively, lower) frequencies as shown in Fig. 6(b), in which the position of the peaks for
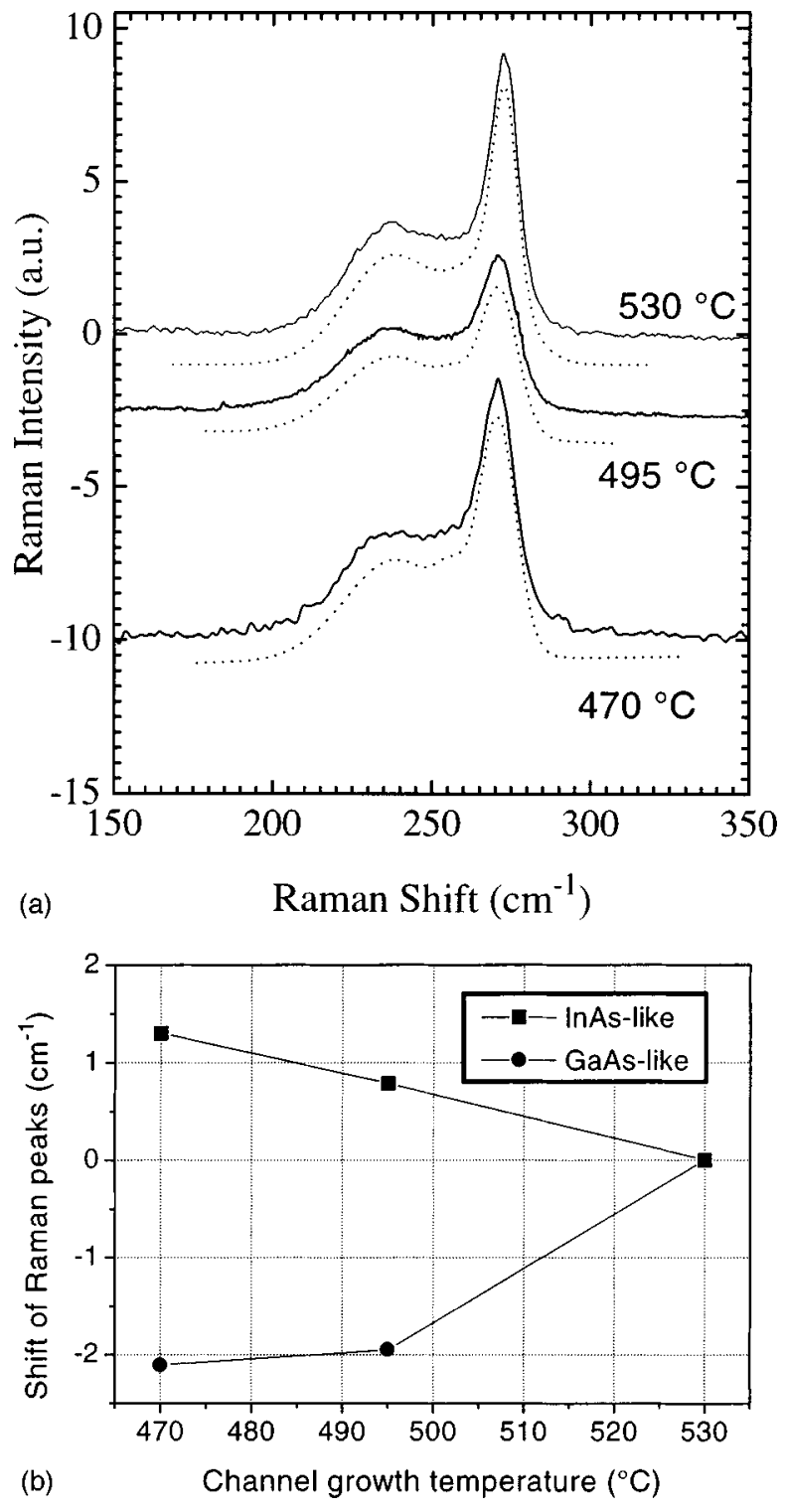

FIG. 6. (a) Raman spectra of the lattice matched InGaAs channel for different growth temperatures. (b) Shift of the main Raman peaks taking as origin the frequency of the InAs-like peak and GaAs-like peak of the lattice matched channel grown at $530{ }^{\circ} \mathrm{C}$.

the sample grown at $530{ }^{\circ} \mathrm{C}$ has been taken as the $y$-axis origin. In addition, as growth temperature decreases, the InAs-like peak is less resolved which is indicative that the composition is not well defined, as required if lateral modulations of composition are present, as recently suggested by Mintairov et al. ${ }^{8}$

\section{B. Surface corrugation in lattice mismatched samples}

In the case of lattice mismatched samples, XTEM observations reveal a three-dimensional growth mode of the strained well [Fig. 7(a)]. Furthermore, this undulation is not induced by a decomposition of the InGaAs subchannel, since the channel layer appears perfectly homogeneous. Lateral domains are not observed at all, neither for $g=220$ nor for 


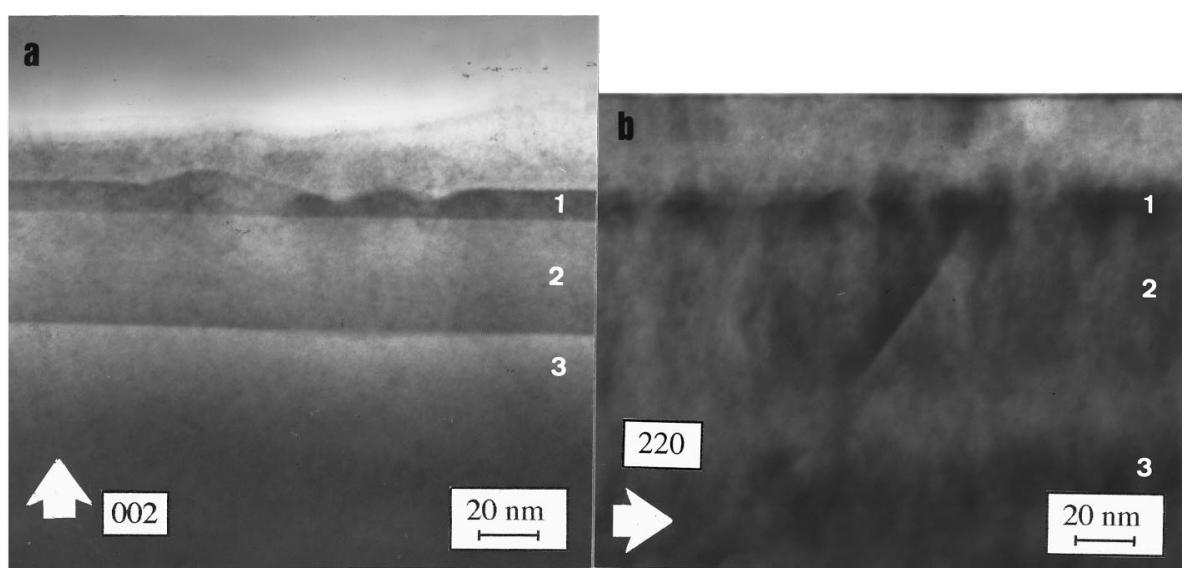

FIG. 7. Cross sections along [ $1 \overline{1} 0]$ of the samples with lattice mismatched channel grown at $520{ }^{\circ} \mathrm{C}$, obtained with (a) $g=002$ and (b) $g=220$. The numbers correspond to: the lattice mismatched InGaAs channel (1), the lattice matched InGaAs subchannel (2), and the InAlAs buffer layer (3).

$g=002$. We can compare Figs. 7 and 5 to notice the absence of lateral domains in these lattice mismatched layers. These results indicate that, in the presence of high lattice mismatch, the alloy decomposition does not occur, even at low $T_{g}$, demonstrating the fact that the pseudomorphic growth of strained layers may stabilize alloys otherwise subjected to alloy spinodal decomposition. ${ }^{7}$ Furthermore, comparing Figs. 4(a), 4(b), and 4(c) we notice that high $T_{g}$ increases the strength of 3D growth, preferentially observed around the square-shaped coarse pattern or the complex defects. In previous works ${ }^{1,4}$ we have shown that both, coarse pattern and defects, originate mainly at the InAlAs buffer layer/InP interface and propagate through the structure. The results also confirm that the contrast pattern is related to the presence of composition inhomogeneities in the buffer layer induced by In segregation at high temperature, which propagates through the whole structure. As proposed in Ref. 1, at low $T_{g}$, the propagation of these buffer layer inhomogeneities stops at the channel buffer interface. However, as $T_{g}$ increases they could propagate inside the InGaAs channel, favoring the $3 \mathrm{D}$ growth mode and leading to the reduction of $\mu$ values.

\section{CONCLUSIONS}

The authors have shown that the degradation and anisotropy of Hall mobilities observed in HEMT structures with InGaAs channel grown at different temperatures are related to the evolution of a surface corrugation due to: alloy decomposition in lattice matched channels favored at low $T_{g}$ or strain induced in lattice mismatched channels favored at high $T_{g}$.

${ }^{1}$ F. Peiró, A. Cornet, J. R. Morante, and A. Georgakilas, J. Vac. Sci. Technol. B 13, 1006 (1995).

${ }^{2}$ M. Beck and M. Ilegems, Proceedings of 8th IPRM Conference, Germany, 1996, p. 97, IEEE Catalog 96 CH 35930.

${ }^{3}$ F. Peiró, J. C. Ferrer, A. Cornet, J. R. Morante, M. Beck, and M. A. Py, Inst. Phys. Conf. Ser. 160, 491 (1997).

${ }^{4}$ F. Peiró, J. C. Ferrer, A. Cornet, J. R. Morante, M. Beck, and M. A. Py, J. Vac. Sci. Technol. B 15, 1715 (1997).

${ }^{5}$ K. C. Hsieh, J. N. Baillargeon, and K. Y. Cheng, Appl. Phys. Lett. 57, 2244 (1990)

${ }^{6}$ F. Peiró, A. Cornet, J. R. Morante, and A. Georgakilas, Mater. Res. Soc. Symp. Proc. 417, 265 (1996).

${ }^{7}$ F. Glas, J. Appl. Phys. 62, 3201 (1987).

${ }^{8}$ A. M. Mintiarov and H. Temkin, Phys. Rev. B 55, 5117 (1997). 